\title{
REMARQUES SUR LA TRADUCTION DE LA POESIE
}

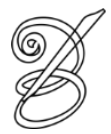 \\ Inês Oseki-Dépré \\ (Professeur émérite Université d'Aix-Marseille-France) \\ inesoseki@gmail.com
}

\begin{abstract}
Résumé: S'il y a un critère pour définir l'objet "poésie", il est, à mon avis contenu dans la traduction de la poésie. En effet, selon qu'un texte poétique est ou non "traduisible", on peut en distinguer trois catégories: le texte est intraduisible; le texte est littéralement traduisible; le texte est traduisible moyennant des transformations dans le but d'obtenir une traduction "isomomorphe".Ceci nous amène à une autre considération: la poésie s'inscrit dans le langage, certes, mais surtout dans une langue, dans sa langue et, de ce fait, lorsqu'elle est transposée dans une autre langue, des problèmes vont apparaître. Enfin, il s'agit dans le présent article de rendre rapidement compte de l'état de la poésie contemporaine en France. D'où la conclusion: la poésie est universelle mais pas toujours.
\end{abstract}

Mots clés:Traduction, traduction littérale ou "source oriented", traduction libre ou "target oriented", poesie contemporaine, novlangue, traductibilite, intraductibilité, intentio operis, intentio auctoris.

Resumo: Se há um critério para definir o objeto "poesia", ele está, em minha opinião, contido na tradução de poesia. Com efeito, conforme o texto poético seja ou não "traduzível”, pode-se distinguir três categorias: o texto intraduzível; o texto é literalmente traduzível; o texto é traduzível mediante transformações com o fito de obterse uma tradução "isomórfica". Isso nos leva a outra consideração: a poesia se inscreve na linguagem, claro, mas, sobretudo, numa língua, na sua língua, e, em decorrência disso, quando é transportada noutra língua, surgirão problemas. Enfim, trata-se no presente artigo de dar rapidamente conta do estado da poesia contemporânea na França. Donde a conclusão: a poesia é universal, mas nem sempre.

Palavras-chave: tradução literária ou "source oriented", tradução libre ou "target oriented", poesia contemporânea, novlangue, tradutibilidade, intradutibilidade, intentio operis, intentio auctoris.

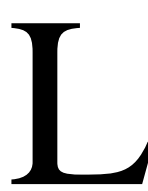

a traduction de la poésie apparaît comme un moyen de dégager non seulement une méthode qui permettrait d'effectuer des re-créations poétiques (ou transcréations) mais également d'identifier quelques unes des caractéristiques de la poésie contemporaine.

J'aimerais, à ce propos, évoquer dans un premier temps la notion d'isomorphisme, chère à Haroldo de Campos, grand poète, essayiste et traducteur brésilien. Dans un second temps, j'évoquerai la question de la pratique de la traduction de poésie, et enfin, les questions relatives à la langue en rapport avec les différentes poétiques dans lesquelles elle se manifeste. Ce faisant, il se dégagera une image aporétique de la poésie oscillant entre l'universel et le singulier. 
Selon Haroldo de Campos, la traduction de la poésie doit s'appuyer sur le principe d'isomorphisme pour que le texte traduit soit en conformité avec l'original, voire s'y substitue en langue d'arrivée.

L'isomorphisme, on le sait, est une notion empruntée à la mathématique. ${ }^{1}$ Transposé sur la traduction littéraire. Selon Haroldo de Campos, il s'agit de:

\begin{abstract}
Traduire la forme, en d'autres termes, le "mode d'intentionalité" (Art des Meinens) d'une œuvre - une forme signifiante, par conséquent, intracode sémiotique signifie, en termes opérationnels d'une pragmatique du traduire, refaire le parcours configurateur de la fonction poétique, en le reconnaissant dans le texte de départ et en le re-inscrivant, en tant que dispositif d'engendrement textuel, dans la langue du traducteur, pour aboutir au poème trans-créé comme projet isomorphique du poème original (CAMPOS, 1981, p. 181).
\end{abstract}

Autrement dit, produire une traduction "isomorphique", où la "relation intime et réciproque entre les langues" (Benjamin) vise à "dés-occulter... sous la couleur d'une 'affinité élective', leur forme sémiotique essentielle" (CAMPOS, 1981, p. 189) qui n'implique pas, pour la poésie, des restrictions métriques "jeu parcimonieux des rimes terminales et la compulsion métrique" (CAMPOS, 1981, p. 189).

Le point de départ de l'opération traduisante est donc la description (formulée ou nonformulée) de l'objet à traduire, c'est-à-dire le poème original.

Le présupposé d'Haroldo de Campos est que le poème original figure ce que Roman Jakobson appelle la fonction poétique quant à la matérialité du signifiant et quant à la projection de l'axe paradigmatique sur l'axe syntagmatique.

C'est donc en "démontant" la machine du poème et la remontant ensuite, qu'Haroldo de Campos a produit de magnifiques traductions de Dante, Mallarmé, Pound, Joyce, entre autres.

Je reprends un exemple que j'ai déjà cité dans un ouvrage précédent (OSEKI-DÉPRÉ, 2006). Il s'agit de la traduction de la Canzone de Guido Cavalcanti.

\footnotetext{
CANZONE (Guido Cavalcanti c.1259-1300)

Donna mi prega, - perch'eo voglio dire

D'un accidente - che sovente - è fero

Ed è si altero - ch'é chiamato amore:

Si chi lo nega - possa 'l ver sentire!

Ed a presente - conoscente - chero,

Perch'io no spero - ch'om di basso core

A tal ragione porti canoscenza:
} 
Chè senza - natural dimonstramento

Non ho talento - di voler provare

Là dove posa e chì lo fà creare,

E qual sia sua vertute e sua potenza

L'essenza - poi e ciascun suo movimento,

É 'l piacimento - che'l fà dire amare,

E s'omo per veder lo pó mostrare

(...) (CAVALCANTI, 1967, p. 47, extraits).

La traduction de Haroldo de Campos ${ }^{2}$ (1970), poète contemporain, maintient comme Ezra Pound, autre traducteur de Cavalcanti, un langage à la fois archaïsant et moderne en plus de la rime et de la cadence qui, comme on l'a signalé, sont pour le poète américain $^{3}$ les vecteurs de l'émotion.

Pediu-me uma Senhora

fale agora

Dum acidente

geralmente

forte

E de tal porte

que é chamado Amor

Quem ora o nega

prove-o novamente

Mas um presente

entendedor

requeiro

Nem espero

de um baixo coração

Conhecimento aberto desta razão

Se não se apega

a natural sustento

Meu intento não

vai poder provar

Onde êle nasce e quem o faz criar

Qual é sua virtude e sua potência

A essência

e depois o movimento

O encantamento

que há em dizer amar

E se alguém pode vê-lo à luz do olhar ${ }^{4}$ 
Dans le poème original, les rimes sont fréquemment extérieures, initialement en terza rima: v.1/ v.4; v.2/v.5; v.3/v.6, alternant avec des rimes intérieures (canoscenza (v.7) - chè senza (v.8); dimostramento (v.8) - non ho talento (v. 9), suivies de deux rimes (riches): provare (v.9)/creare (v. 10). Les deux vers suivants contiennent des rimes intérieures Potenza (v.11) - L'essenza (v.12); movimento (v. 12) - piciamento (v.13) et le poème se termine sur deux vers aux rimes plates (distique): amare, mostrare.

Or, à la lecture du poème brésilien, on note des transformations par rapport à l'original. La traduction d'Haroldo de Campos condense le poème de Cavalcanti, qui de 14 devient un poème de 10 vers. Les rimes sont riches, semi-batelées (fin de vers/coupe) senhora/agora; acidente/geralmente; forte/porte; Amorlentendedor; novamentelpresente; requeiro/espero (assonance); sustento/intento, ou plates et le poète ne maintient les rimes finales qu'aux deux derniers vers de manière particulière. Il fragmente ainsi le décasyllabe en vers plus brefs (à l'instar de ses propres poèmes), il supprime, comme Pound, les tirets de la version italienne et crée une respiration différente, avec des "blancs", les pauses, 10 sans ponctuation. Le poème apparaît dans son essence, lapidaire. Peut-on dire qu'il s'agit d'une traduction littérale? Comment justifier ici le principe d'isomorphisme que revendique le poète?

Haroldo de Campos est de ceux pour qui la traduction doit répondre aux trois fonctions énumérées par Ezra Pound : lecture, critique et recréation poétique. La fonction de recréation poétique est d'autant plus relevante qu'elle constitue, à ses yeux, non seulement un instrument pour le poète lui-même mais le moyen le plus adéquat pour la formation d'une culture nationale. ${ }^{5}$

Ainsi, à l'instar d'Ezra Pound, ses traductions couvrent des larges pans de l'histoire littéraire, allant de James Joyce (Finnegans Wake) à Maïakovski; des troubadours aux poètes russes; de Dante (Le Paradis) à Mallarmé; du théâtre No à Octavio Paz et Ezra Pound (Cantares); de la Bible (l'Ecclésiaste, Bere 'shith) à l'Iliade. ${ }^{6}$

La traduction de textes créatifs devient une création parallèle, mais autonome.

Comment? En traduisant le signe lui-même, c'est-à-dire, sa tangibilité, sa matérialité (propriétés sonores, propriétés graphico-visuelles), enfin, tout ce qui forme, pour Charles Morris, l'iconicité du signe esthétique, compris comme "signe iconique" - celui "qui est d'une certaine façon pareil à ce qu'il dénote". ${ }^{7}$ Une certaine forme de littéralité en somme. Mais une littéralité pensée en termes modernes ou comme le dit Jacques Derrida, ${ }^{8}$ la 
traduction "littérale" (ou relévante) serait une traduction qui prendrait place (qui relève) de l'original mais qui le relèverait en même temps.

Dans un second temps, il s'agit de proposer une recréation du texte original "à travers les équivalents dans notre langue de toute l'élaboration formelle (sonore, conceptuelle, imagée)", dans le dessein de parcourir les étapes créatives originales. Ce qui, concrètement, revient à privilégier la forme (allitérations, paronomases, assonances) autant sinon davantage que le concept et qui rejoint les paroles de Pound à propos de sa traduction de Cavalcanti:"The perception of the intellect is given in the word, that of the emotions in the cadence." 9 On pose ici le problème de la langue d'arrivée sur lequel on va revenir plus loin.

La troisième étape haroldéenne, qui correspond à son évolution aussi bien dans son œuvre que dans sa manière de traduire (work in progress), consiste à revendiquer une traduction qui oblitère l'original. Il s'agit en quelque sorte de passer d'une traductionrecréation (T-R) selon Etkind $(1982)^{10}$ à une traduction-imitation (T-I), ce qu'il accomplit dans sa "translucifération" du Faust de Goethe (1981). ${ }^{11}$

Haroldo de Campos a proposé plusieurs essais pour indiquer sa façon poundienne (make it new) de traduire, dont l'un, où il évoque sa traduction du "Coup de dés" de Stéphane Mallarmé, présente sa "méthode" transcréatrice en plusieurs points:

- La lecture commence par s'intéresser au niveau graphique (typographique) de l'original pour laquelle l'œuvre de Robert Greer Cohn, L'œuvre de Mallarmé (Paris, Les Lettres, 1951) lui sert de guide;

- Sur le même plan, sur la base de la recherche peircienne, le traducteur examine le graphe numérologique qui consiste en la répétition calculée de mots. Pour Mallarmé, telle clé est représentée par le chiffre sept, anagrammatisé dans EXCEPTÉ/ PEUT-ÊTRE, qui est le nombre de formes verbales gérondives, correspondant aux sept étoiles de la constellation de l'Ourse Majeure. Il en tient compte et les traduit quasiment toutes par la forme gérondive. Il fait remarquer que le vers final contient sept mots ("Toute pensée émet un Coup de Dés"), de même que les vers précédents.

- En même temps, note-t-il l'importance de la "reprise étymologique", par exemple pour le mot "vers", polysémique, qui désigne le vers du poème et la direction vers laquelle il s'achemine.

- Au niveau de la grande articulation macro-syntaxique, il faudra faire remarquer le problème du JAMAIS, dans la phrase-titre qui, composée en majuscules, articule le poème, en le parcourant, de façon espacée, provoquant de nouveaux dessins. 
- Les correspondances sémantico-visuelles doivent être lues sur l'axe horizontal et vertical, comme des notes harmoniques. Ainsi, le vers:

naguères d'où sursauta son délire jusqu'à une cime flétrie où Greer Cohn décèle en ‘jus' (de jusqu'à) l'élément "sève", associé à "flétrie", est rendu par: de onde há pouco sobressaltara seu deLÍRIO a um cimo fenescido (où la graphie de "fenescido" peut renvoyer à "fênix-sido", avec l'idée de résurrection.)

Il est difficile pour un traducteur de prétendre affirmer réaliser des traductions "littérales" ou des traductions "littéraires". La dichotomie traditionnelle établie par nombre de linguistes ou philosophes du langage n'est en fait qu'opérationnelle: d'un côté, on dira que la traduction et "source oriented", tournée vers la langue de départ, ce sont les premières, de l'autre qu'elle est "target oriented", tournée vers la langue d'arrivée et ce seraient les dernières. ${ }^{12}$ Antoine Berman, ${ }^{13}$ à la suite de Walter Benjamin et son fameux essai "La Tâche du traducteur", 14 milite pour une traduction littérale, éthique, et parmi les traducteurs paradigmatiques de ce courant, il cite entre autres Klossowski (et sa traduction de l'Enéide ${ }^{15}$ ), et Hölderlin (et sa traduction d'Antigone). ${ }^{16}$ Dans le premier cas, si littéralité y est, elle est plutôt "mimétique" latine; dans le second, grecque. Dans les deux cas, ce n'est pas tant le texte qui est traduit littéralement mais la langue du texte original...

Pour n'en rester qu'à l'œuvre d'Haroldo de Campos, dont j'ai traduit plusieurs textes, de différentes périodes, de différentes factures, si j'essaye de pratiquer la méthode “isomorphique", la façon de traduire varie selon le poème à traduire. Je pense que c'est le cas de la majorité des traductions.

Le principe à respecter est simple: la littéralité doit respecter la littérarité du départ, autrement dit, les transformations opérées lors du processus traductif ne doivent pas altérer la littérarité de l'original. Je propose ici un exemple: Galaxies, fragment $45 .{ }^{17}$

\footnotetext{
mais uma vez junto ao mar polifluxbórboro polivozbárbaro polúphloisbos polyfizzboisterous weitaufrauschend fluctissonante esse mar esse mar esse mar texto por quem os signos dobram marujando num estuário de papel num mortuário num monstruário de papel murmur-rumor-remurmunhante escribalbuciando você converte estes signos-sinos num dobre numa dobra de finados enfim nada de papel estes signos você os ergue contra tuas ruínas ou tuas ruínas contra estes signos balbucilente sololetreando a sóbrio neste eldorido feldorado latinoamargo tua barrouca moropopéia ibericaña...
} 
En français:

une fois de plus vers la mer polifluxborborante polivocobarbare polúphloisbos polyfizzboisterous weitaufrauschend fluctissonante vers la mer vers la mer* mertexte pour qui consonnent les glas cette mer matelotante dans son* extuaire de papier mortuairemonstruaire de papier murmur-rumeur-ruante* toi *scribalbutiant tu convertis ces signes qui sonnent en chant en plein chant* de jourdesmorts* funiveresse du papier ces signes tu les dresses contre tes ruines ou tes ruines contre ces signes-sons* balbusiencieux sololettriste* syllabant* dans ton eldouloureux fieldorado latinoamer ta barauque morépopée ibericaña... ${ }^{18}$

On pourra remarquer des modifications (signalées par un*) par rapport à l'original: la plupart ayant comme buts, premièrement le maintien du rythme original (sonorités, allitérations, accents: ver la mer, plutôt que "cette mer"; "qui sonnent en chant en plein chant" remplace "sinos (cloches)-signos", qui est re-introduit en "signes-sons"; "sololettriste", motvalise, remplace avec bonheur "sololetreando" qui signifierait 'épelant en solo'); deuxièmement la lisibilité en langue française ("son extuaire" à la place de "un extuaire"). Ici, l'intention du traducteur est de produire une traduction qui rende les mêmes effets que l'original.

Dans d'autres occasions, j'ai traduit bien plus "littéralement", lorsque cela était possible. Mais il est arrivé que je sois obligée, si le rythme et les sonorités étaient visiblement prioritaires dans 1'intentio operis, de modifier considérablement l'original tout en maintenant ses caractéristiques prosodiques et sémantiques, comme dans le poème "Forme de faim" du même poète ${ }^{19}$ où les assonances jouent le rôle de rimes: substitution de "prata/preta" (argent/noire) par "ivoire/noire"; déplacement du mot "vil”" au vers précédent ("iris/vill”) pour le maintien du son /i/ alors que dans l'original on trouve "anil/vil" qui en français devient "anil/nulle" et ainsi de suite. Le traducteur a donc un triple rôle: critique, traducteur et créateur, il est poéticien et poète à la fois. Il doit par conséquent avant de traduire, identifier non seulement la forme, mais le caractère du poème à traduire: parfois l'original se contente d'une simple transposition avec des légères interventions du traducteur (choix du lexique, ordre des mots), parfois il exige que le traducteur mobilise toutes les ressources de la langue d'arrivée, ce qui lui permet de se rendre compte de la variété de formes et procédés du départ (intertextualité, collage, adresse à un destinataire, narrativité, fragmentation, emprunt, accent sur la syntaxe, sur les sonorités...) et également d'identifier le "genre" utilisé: la prose; le vers libre; le fragment; le silence, pas toujours; la forme brève pas toujours. Mais dans tous les cas, le traducteur pratique une forme de littéralité qui tienne compte du littéraire. 
Ce qui nous amène au dernier point de notre développement: on peut, en effet, penser qu' une telle méthode traductive, telle qu'on l'a exposée ci-dessus - attentive à la forme peut s'appliquer à la traduction de la poésie en général, ce qui n'est que partiellement vrai. Car, contrairement à la doxa, la poésie est peut-être universelle mais pas toujours et cela se vérifie lors de l'épreuve de la traduction. De plus, les exemples convoqués ici renvoient à une intention poétique (forme) explicite, celle qui se donne à voir sans ambigüité comme poésie.

En traduction, cela est particulièrement notable et on peut quasiment classer les poèmes à traduire sous trois catégories parmi lesquels ceux qui se laissent traduire, ceux qui ne se laissent pas traduire, ceux qui se laissent traduire au moyen de grandes transformations.

Un poème peut ne pas être traduisible non pas parce qu'il est intrinsèquement "intraduisible", comme on dit des poèmes de Celan (qui ont été souvent traduits), par exemple, mais parce que lorsqu'il se retrouve dans une autre langue, il perd pour ainsi dire sa consistance de poème. Ou alors, tout en ayant la forme d'un poème, il apparaît comme un objet "tombé du ciel", tant sa réception est liée au projet initial.

Par ailleurs, un poème qui se laisse traduire est en général un poème qui "tient", dont les parties sont suffisamment structurées (même s'il est fait de peu de mots), ou bien du fait que l'auteur a réussi à lui imposer une marque cohérente (un style).

Ce qui soulève immédiatement le problème de la langue, qu'elle soit celle du départ ou de l'arrivée, entendue comme langue à usage poétique, historique, ce qui englobe, bien sûr, les connotations.

Ainsi, si l'on se réfère, par exemple, à ce qui s'appelle "l'extrême contemporain" français (post-moderne?), héritier de la Modernité, deux points se font remarquer immédiatement:

- le premier que l'on peut appeler "la crise du vers", depuis Mallarmé, qui a comme conséquence, et non des moindres, que on ne traduise pas les rimes en français (note les troubadours) dans la mesure où une traduction contemporaine en vers rimées serait perçue comme anachronique. Or, souvent, ce sont des poètes qui traduisent et, de ce fait, ils s'adaptent aux "canons" de la contemporanéité française;

- le second, le poids de la tradition poétique française, en quelque sorte lié à cette crise, qui est un problème lié au contexte de formation de la littérature française.

Dès lors, depuis Mallarmé et, malgré "la vieillesse d'Alexandre", 20 analysée par Jacques Roubaud, non seulement il est proscrit d'utiliser la rime dans la poésie française contemporaine, mais on poursuit une sorte d'agone contre la tradition. ${ }^{21}$ Francis Ponge, les avant-gardes, toute la poésie d'aujourd'hui tend vers le "nouveau", mais vers un nouveau- 
nouveau, si l'on peut dire, soit, cette "exploration solitaire qui conduit à une grande diversité", selon Joëlle Gardes. ${ }^{22}$ Cette situation est loin d'être universelle et, justement, elle place le problème d'une intraduisibilité d'un autre ordre: en admettant que les poèmes seraient linguistiquement traduisibles (sauf pour le cas de quelques poèmes oulipiens), leur "combat" ne serait pas perceptible dans un contexte différent. En somme, l'intentio auctoris peut conditionner la réception de l'intentio operis.

Dans son ouvrage Salut les modernes/Salut les anciens, paru déjà en 2000, Christian Prigeant, poète et théoricien français contemporain, dresse un tableau de la situation poétique de l'extrême contemporain et, pour ce faire, évoque la novlangue qui semble s'être imposée dans nos sociétés: “une langue imposée, dé-syntaxisée, non connotante, dont l'objectif serait de "rendre impossible tout autre mode de pensée", ce qui en soi représente une raison d'écrire (ou de "mécrire")."23

Le problème évoqué ici semble être celui de ce que Pierre Bourdieu entre autres ${ }^{24}$ appelle la langue "dominante" (la belle langue légitime) qui touche aussi la traduction et dont le principal exemple est représenté par les "belles infidèles", mais qui perdure jusqu'aujourd'hui. La langue "standard" ou la langue dite littéraire.

Christian Prigeant s'efforce de caractériser ce "mécrire", d'en donner des exemples. Il évoque Christophe Tarkos et sa pâte-mot (patmo) dont le travail consisterait à déborder l'espace du sens. Un peu, dit-il, comme faisait jadis Burroughs et son cut-up, avec des prélèvements et des recyclages. D'où il s'entrevoit “à nouveau l'enjeu d'écrire": la question "de ce que la langue fait des sujets." 25

La poésie dans ce cas, comme dans le cas du "déchet de l'expérience" proustien, est résistance.

La question du "comment faire" semble alors se déplacer vers le "pour quoi faire": inventer le négatif, déjouer la novlangue, faire du nouveau au moyen du "le ready-made pince-sans-rire, la distanciation neutralisée, la prosodie atone, le refus, en tout cas, de toute pression de l'expressivité" (PRIGENT, 2000, p. 20).

On est loin de Francis Ponge qui, tout en évitant la "pression de l'expressivité", tout en prenant des distances en parlant des "choses", dans le même mouvement "contre" la langue, articule, formule, est encore dans un discours stylé, reconnaissable.

Les contemporains, cela étant, ne sont pas très loin de Baudelaire: leurs moyens semblent proches des suggestions baudelairiennes: le gestes (sonorisés, dessinés, peints, performarisés), les regards (photo, cinéma, télévision, corps et visages saisis), les ports 
(attitudes). La modernité d'aujourd'hui n'est pas, loin s'en faut, l'actualité, la médiatisation. Bref, l'extrême contemporain serait dans la continuité de "l'innommable".

La poésie serait alors "l'élévation de cela à la conscience: formalisation des moments, l'assomption du réel en langues en tant que ces moments sont, simultanément, moments d'effondrement des langues face à la montée en elles de la puissance défigurante du réel innommable" (PRIGENT, 2000, p. 29). La poésie ainsi, ouvre le monde.

Prigent soutient que la littérature est "l'expérience des limites" et, saluant Artaud et Bataille, pose la question: "la littérature est-elle jamais autre chose qu'une mise en jeu du sujet dans sa langue en tant que cette mise en jeu joue le sujet là où la langue le limite?" (PRIGENT, 2000, p. 36).

Il cite des revues dont Formules, dans la tradition du formalisme oulipien. Pour ces derniers, dont les représentants seraient Jacques Roubaud, Jacques Jouet, Michelle Grangaud..., la "modernité vraie résiderait dans le 'calcul conscient comme moteur de l'écriture'" à l'instar de Poe, Roussel.

En quelques mots, les procédés poétiques contemporains pourraient se résumer en:

1) le refus de l'emphase sur l'auteur, de la métaphore, le refus du pathos lyrique, des bouffées d'intériorité sensible, des spéculations métaphysiques (PRIGENT, 2000, p. 50), peu d'intérêt pour le partage précodé des genres (les poètes contemporains sont aussi peu classables que Guyotat, Novarina, Lucot). Certains opteraient pour la poésie (vers, prosodie), d'autres pour la prose (avec réduction ou épaississement);

2) l'élimination de la verticalité au profit d'une horizontalité; poésie de surface, "faciale" sans profondeur, sans “secret”, littérale. On est loin de la fonction poétique jakobsonienne.

La traduction poétique se pose alors comme un cas de "translation", à savoir, une traduction accompagnée d'un commentaire, d'une explicitation contextuelle, voire méta-linguistique. ${ }^{26}$ Ce qui tendrait à conforter l'idée que la poésie, tout en étant une expérience singulière, à caractère universel, est liée à son contexte, son époque, sa langue et qu'en définitive, la traduction, comme la réception, a son heure. 


\section{RÉFÉRENCES BIBLIOGRAPHIQUES}

BENJAMIN, Walter. La tache du traducteur. In: Mythe et violence. Traduction de M. de Gandillac. Paris: Dénoël, 1971.

BERMAN, Antoine. Hölderlin, ou la traduction comme manifestation. In: Les Tours de Babel. Paris: T.E.R, 1985b. p. 93-108.

l'epreuve de l'etranger. Paris: Gallimard, 1967-1980.

Les Tours de Babel. Paris: T.E.R, 1985a.

1995.

Pour une critique des traductions: John Donne. Paris: Gallimard,

BOURDIEU, Pierre. Ce que parler veut dire. Paris: Fayard, 1982.

CAMPOS, Haroldo de. Deus e o Diabo no Fausto de Goethe. São Paulo: Perspectiva, 1981.

courante, 1998.

. Galaxies. Traduction de Inês Oseki-Dépré. La Souterraine: Ed. La Main

Paris: Éditions Al Dante, 2005.

Haroldo de Campos, une anthologie. Traduction de Inês Oseki-Dépré. Traduzir e trovar. São Paulo: Edições Papyrus, 1968.

CAVALCANTI, Guido (a cura di). Rima. Turin: Einaudi, 1967.

DERRIDA, Jacques. Qu'est-ce qu'une traduction relevante? In: QUINZIÈMES ASSISES DE LA TRADUCTION LITTERAIRE (Arles 1998), Arles, Actes Sud, 1999.

ETKIND, E. Un art en crise. Essai de poétique de la traduction poétique. Lausanne: L'Âge d'Homme, 1992.

OSEKI-DÉPRÉ, Inês (1990). Théories et pratiques de la traduction littéraire. $2^{\text {ème }}$ éd. Paris: Armand Colin, 2006.

OSEKI-DÉPRÉ, Inês. "D'une traduction amnésique", à propos de la traduction brésilienne de Quelque chose noir, de Jacques Roubaud où ce qui est problématique n'est pas le texte à traduire mais sa réception hors tradition. Alea, Revue d'Études Néo-Latines, Rio de Janeiro, 2011.

PRIGENT, Christian. Salut les anciens/Salut les modernes. Paris: P.O.L., 2000.

ROUBAUD, Jacques. La vieillesse d'Alexandre. Paris: François Maspero, 1978.

\footnotetext{
${ }^{1}$ En mathématiques, un isomorphisme entre deux ensembles structurés est une application bijective qui préserve la structure et dont la réciproque préserve aussi la structure (Wikipédia).

${ }^{2}$ Selon Jacques Roubaud supérieure à la première, conférence à Yale, octobre 1999.

${ }^{3}$ On peut remarquer que dans certaines littératures, si la rime n'est plus utilisée par les poètes, elle est maintenue pour la traduction du vers rimé. C'est le cas en général des littératures ibériques (lusophones et hispanophones).

${ }^{4}$ Voir OSEKI-DÉPRÉ, 2006, p. 123.
} 
${ }^{5} \mathrm{Si}$ on se réfère à ce que le poète appelle son "paideuma", qui serait à la base d'une poésie innovante (la poésie
concrète), on pourra constater que le nombre d'auteurs traduits est impressionnant et que les traductions ont été à la base d'un renouveau de la poésie nationale.

${ }^{6}$ Partisan du "laboratoire de traductions", plusieurs de ses traductions ont été faites avec son frère Augusto de Campos, Décio Pignatari et autres poètes.

${ }^{7}$ Voir CAMPOS, 1981.

${ }^{8}$ Voir DERRIDA, 1999.

${ }^{9}$ On lui connaît toutefois des traductions-détour pour les poèmes chinois, à l'instar de Pound.

${ }^{10}$ ETKIND, 1992, p. 25.

${ }^{11}$ Voir Inês Oseki-Dépré, "Haroldo de Campos: de l'anthropophagie à la translucifération", conférence prononcée aux rencontres Europalia, Paris, Sorbonne, 2011.

${ }^{12}$ On peut trouver l'affirmation de cette dichotomie aussi bien chez Humboldt, que chez Georges Mounin, JeanRené Ladmiral, Umberto Eco, Even-Zohar: elle ne recouvre qu'un aspect de la question, celui des traductions "classiques". Voir OSEKI-DÉPRÉ, 2006, p. 73-75.

${ }^{13}$ Voir BERMAN, 1967-1980; BERMAN, 1985a; et BERMAN, 1995.

${ }^{14}$ BENJAMIN, 1971; traduction Martine Broda, in Po\&sie, n. 55, $1^{\text {er }}$ trimestre 1991. La question est plus complexe qu'il n'y parait.

${ }^{15}$ Voir l'Enéide, Marseille, traduction de Pierre Klossowski, Ed. André Dimanche, 1989.

${ }^{16}$ Voir BERMAN, 1985b.

${ }^{17}$ Voir CAMPOS, 1998.

$18{ }^{18}$ L'ensemble de poèmes des Galaxies, traduits sur une longue durée, ont été minutieusement contrôlés par le poète lui-même.

${ }^{19}$ Voir de CAMPOS, 2005, p. 124.

${ }^{20}$ Voir ROUBAUD, 1978.

${ }^{21}$ Ce qui entraine comme conséquence la traduction des troubadours gallego-portugais en vers libre, ce qui enlève aux trovas leur principe fondateur.

${ }^{22}$ J'emprunte cette expression à Joëlle Gardes-Tamine, in "Remarques sur la poésie contemporaine”, article paru en 2004.

${ }^{23}$ Voir PRIGENT, 2000, p. 16.

${ }^{24}$ Voir BOURDIEU, 1982.

${ }^{25}$ Voir PRIGENT, 2000, p. 17.

${ }^{26}$ Voir OSEKI-DÉPRÉ, 2011, p. 387 et seq. 